\title{
Deciphering genes associated with diffuse large B-cell lymphoma with lymphomatous effusions: A mutational accumulation scoring approach
}

Sina Abdollahi ${ }^{1}$, Seyedeh Zahra Dehghanian ${ }^{2 \dagger}$, Liang-Yi Hung ${ }^{3,4,5,6,7 \dagger}$, Shiang-Jie Yang ${ }^{8 \dagger}$, Dao-Peng Chen ${ }^{9 \dagger}$, L. Jeffrey Medeiros ${ }^{10}$, Jung-Hsien Chiang ${ }^{1,11^{*}}$ and Kung-Chao Chang ${ }^{12,13,14,15^{*}}$

\begin{abstract}
Introduction: Earlier studies have shown that lymphomatous effusions in patients with diffuse large B-cell lymphoma (DLBCL) are associated with a very poor prognosis, even worse than for non-effusion-associated patients with stage IV disease. We hypothesized that certain genetic abnormalities were associated with lymphomatous effusions, which would help to identify related pathways, oncogenic mechanisms, and therapeutic targets.

Methods: We compared whole-exome sequencing on DLBCL samples involving solid organs $(n=22)$ and involving effusions $(n=9)$. We designed a mutational accumulation-based approach to score each gene and used mutation interpreters to identify candidate pathogenic genes associated with lymphomatous effusions. Moreover, we performed gene-set enrichment analysis from a microarray comparison of effusion-associated versus non-effusion-associated DLBCL cases to extract the related pathways.

Results: We found that genes involved in identified pathways or with high accumulation scores in the effusion-based DLBCL cases were associated with migration/invasion. We validated expression of 8 selected genes in $D L B C L$ cell lines and clinical samples: MUC4, SLC35G6, TP53BP2, ARAP3, IL13RA1, PDIA4, HDAC1 and MDM2, and validated expression of 3 proteins (MUC4, HDAC1 and MDM2) in an independent cohort of DLBCL cases with $(n=31)$ and without $(n=20)$ lymphomatous effusions. We found that overexpression of HDAC1 and MDM2 correlated with the presence of lymphomatous effusions, and HDAC1 overexpression was associated with the poorest prognosis.
\end{abstract}

Conclusion: Our findings suggest that DLBCL associated with lymphomatous effusions may be associated mechanistically with TP53-MDM2 pathway and HDAC-related chromatin remodeling mechanisms.

Keywords: Diffuse large B-cell lymphoma, Lymphomatous effusions, Whole exome, Sequencing, Bioinformatics, HDAC1, Prognosis

\footnotetext{
* Correspondence: jchiang@mail.ncku.edu.tw; changkc@mail.ncku.edu.tw ${ }^{1}$ Intelligent Information Retrieval Lab, Department of Computer Science and Information Engineering, National Cheng Kung University, 701 Tainan,

Taiwan

${ }^{12}$ Department of Pathology, College of Medicine, National Cheng Kung

University Hospital, National Cheng Kung University, 138 Sheng-Li Road, 704 Tainan, Taiwan

Full list of author information is available at the end of the article
}

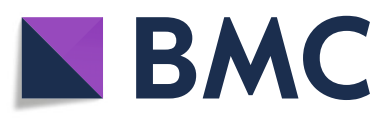

(c) The Author(s). 2021 Open Access This article is licensed under a Creative Commons Attribution 4.0 International License, which permits use, sharing, adaptation, distribution and reproduction in any medium or format, as long as you give appropriate credit to the original author(s) and the source, provide a link to the Creative Commons licence, and indicate if changes were made. The images or other third party material in this article are included in the article's Creative Commons licence, unless indicated otherwise in a credit line to the material. If material is not included in the article's Creative Commons licence and your intended use is not permitted by statutory regulation or exceeds the permitted use, you will need to obtain permission directly from the copyright holder. To view a copy of this licence, visit http://creativecommons.org/licenses/by/4.0/. The Creative Commons Public Domain Dedication waiver (http://creativecommons.org/publicdomain/zero/1.0/) applies to the data made available in this article, unless otherwise stated in a credit line to the data. 


\section{Introduction}

Diffuse large B-cell lymphoma (DLBCL) is the most common form of aggressive lymphoma, accounting for approximately $40 \%$ of all lymphoma cases in Taiwan [1], and about $33 \%$ of cases worldwide. The outcomes of DLBCL patients are variable and therefore stratification into low-risk and high-risk groups is helpful for planning therapy. The development of body cavity effusions in DLBCL is often an indication of a significant pathologic process [2], and previous studies have shown the adverse impact of malignant lymphoma-associated effusions (direct infiltration or distant metastasis) on survival in DLBCL patients $[3,4]$. Others have reported that DLBCL patients with tumorous effusions bear an even worse prognosis than patients with stage IV disease without effusions [4]. The serous fluids in body cavities circulate between parietal and visceral vessels; hence, the development of lymphoma cells in body fluids highlights the growing capability of the lymphoma cells to acquire an aggressive metastatic repertoire, including the ability to migrate, invade, and proliferate.

Conventionally, the identification of cancer behavior and cancer subtypes is attained by utilizing features derived from gene expression. With the advent of wholegenome sequencing and whole-exome sequencing, the list of biological features for identifying cancer behavior has been significantly expanded. In many studies, others have integrated whole-exome sequencing data of various DLBCL biopsy samples to distinguish genetic subtypes of DLBCL $[5,6]$. In addition, several next-generation sequencing (NGS)-based studies have been published deciphering the genetic mutation profile of DLBCL subtypes [7-9]. However, the molecular mechanism underlying the emergence of lymphomatous effusions in DLBCL is largely unknown.

The accumulation of somatic mutations plays a critical role in cancer progression [10]. One of the most informative feature sets to extract the mutational signatures of different cancer subtypes is the regional mutation density (RMD) [11]. Zhang et al. calculated RMD (i.e., the number of mutations per thousand base pairs per patient) to investigate its association with different cancer subtypes. They found that genome-wide RMD profiles represent distinct patterns between melanoma and breast cancer subtypes [10]. Others have shown connections between mutation variations in megabase scale and functional genomic data obtained from tumor cell-oforigin, including chromatin accessibility and replication timing $[12,13]$. Although RMD is one of the widelyused methods for detecting highlighted genes, most mutations are functionally neutral and benign. Only a small number of the mutations, which are called pathogenic, can impair molecular function and consequently lead to cancer [14]. These earlier published findings prompted us to identify the mutation profile underlying the effusion-associated lymphomas in DLBCL.

In this study, we compare the genetic profiles across two groups of patients: those with DLBCL with malignant effusions versus DLBCL patients without effusions (e.g. lymph node-based disease). For this purpose, we performed whole-exome sequencing in 9 effusion-based DLBCL patients and compared them with 22 nodalbased DLBCL samples. Our goal was to identify genes that were highly-mutated in the cases of DLBCL associated with lymphomatous effusions by investigating two factors: mutation accumulation scores and pathogenicity of mutations.

\section{Materials and methods}

The cases of DLBCL associated with malignant effusions were derived from the National Cheng Kung University (NCKU) Hospital and included 9 cases that underwent whole exome sequencing (WES) (Table 1). All samples had a tumor load of more than $80 \%$. The TCGA dataset comprised WES of 22 DLBCL samples with tumors in lymph nodes (https://www.cancer.gov/about-nci/ organization/ccg/research/structural-genomics/tcga).

The study was approved by the institutional review board (NCKUH-A-ER-102-397 and NCKUH-A-ER-105483) and was in accord with the Helsinki Declaration of 1975, as revised in 2013.

\section{DNA extraction and quality control of whole exome sequencing (WES)}

7Libraries were prepared according to Illumina's instructions accompanying the DNA Sample Kit (Part\# 0801 0303). Briefly, DNA was end-repaired using a combination of T4 DNA polymerase, E. coli DNA Pol I large fragment (Klenow polymerase) and T4 polynucleotide kinase. The blunt, phosphorylated ends were treated with Klenow fragment (32 to 52 exo minus) and dATP to yield a protruding 3- 'A' base for ligation of Illumina's adapters which have a single ' $T$ ' base overhang at the 3' end. After adapter ligation DNA was PCR amplified with Illumina primers for 15 cycles and library fragments of $\sim 250 \mathrm{bp}$ (insert plus adaptor and PCR primer sequences) were band isolated from an agarose gel. The purified DNA was captured on an Illumina flow cell for cluster generation. Libraries were sequenced on the Genome Analyzer following the manufacturer's protocols.

\section{Library preparation and sequencing}

For the generation of standard exome capture libraries, we used the Agilent SureSelect XT HS Reagent kit protocol for Illumina Hiseq paired-end sequencing library (catalog\#G9704K). In all cases, the SureSelect XT Clinical Research Exome Version 2 (67.29Mbp) probe 
Table 1 Clinicopathologic features of DLBCL patients with lymphomatous effusions for NGS study

\begin{tabular}{llllllllll}
\hline Case & Age & Sex & $\begin{array}{l}\text { Subtype } \\
\text { COO }\end{array}$ & Genetic & BCL2 & Ki-67 & c-MYC & Stage & Status \\
\hline S9 & 56 & M & ABC & EZB & + & $60-70 \%$ & + & IIIE & Alive \\
S10 & 69 & M & ABC & MCD & - & $60-70 \%$ & + & IIIE & Dead \\
S11 & 71 & M & ABC & EZB & + & $>90 \%$ & + & Dead \\
S12 & 52 & F & GCB & EZB & - & $50-60 \%$ & - & IV & Alive \\
S13 & 45 & M & GCB & N1 & - & $>90 \%$ & + & Alive \\
S14 & 39 & M & GCB & BN2 & + & $70-80 \%$ & - & IV & Alive \\
S15 & 63 & F & GCB & UN & + & $70-80 \%$ & - & IIIE & Dead \\
S16 & 83 & M & GCB & BN2 & + & $80-90 \%$ & + & IVE & Dead \\
S17 & 85 & F & ABC & MCD & + & $>90 \%$ & - & IE & Dead \\
\hline
\end{tabular}

Abbreviations: +, positive; -, negative; Subtype of ABC (activated B-cell) or GCB (germinal center B-cell) determined by Hans cell-of-origin (COO) classification [15]. Genetic subtype based on MCD (MYD88 and CD79B mutations), BN2 (BCL6 fusion and NOTCH2 mutation), N1 (NOTCH1 mutation), and EZB (EZH2 mutation and BCL2 translocation) [6]. All 9 cases had lymphomatous effusions and were negative for Epstein-Barr virus (EBV); Stage, Ann-Arbor staging system

set was used. We used 1 ug genomic DNA to constructed library with Agilent SureSelect XT Reagent kit. The amplification adapter-ligated sample was purified using Agencourt AMPure XP beads (Beckman Coulter, Brea, CA, USA) and analyzed on a TapeStation 4200 D1000 screentape. 500 1000 ng of the gDNA library was prepared for the hybridization with the capture baits, and the sample was hybridized for Agilent hybridization program, captured with the Dynabeads MyOne Streptavidin T1 (Life Technologie, USA) and purified using a Agencourt AMPure XP beads. Use the Agilent protocol to addition of index tags by posthybridization amplification. Finally, all samples were sequenced on Illumina NovaSeq 6000 Sequencer using $150 \mathrm{PE}$ protocol. The bioinformatics analyses and methods for mutation interpreters are detailed in the Supplementary Methods.

\section{Mutation accumulation score}

Since the main purpose of this study was to compare two populations of DLBCL samples, we designed a population-based scoring method by utilizing mutation accumulation across all of the samples. For a given gene, we recorded all mutations that occurred in different samples of the population of interest. In other words, we maintained a list for each mutation that represented the samples hosting the mutation (Supplementary Figure S1A). Each list of a mutation contained $n$ rows, where $n$ represented the number of samples in a population. If a mutation existed in a sample, the sample's corresponding row of the mutation list held the allele frequency of the sample. Otherwise, number $O$ was assigned to the sample's corresponding row in the list. Particularly, $A F_{j, i}$ represented the allele frequency of variant $i$ in sample $j$. Since we considered the mutation accumulation, we took into account the positional distances of the mutations (the number of base pairs between two mutations). Mutations that occurred in a region, bin, of a gene had a similar functional impact compared to mutations in other regions [16-18]. Previously, WinBinVec, a deep learning-based model, revealed that the mutations of a bin have similar functional impact using a onedimensional convolutional neural network [19]. Domanska et al. [20] showed that at small-sized bins, the very high concentration of mutations made the kataegis, localized hypermutation, region stand out clearly. Accordingly, we hypothesized that if a mutation in a sample has a very short distance to another sample's mutation, then they might have a similar functional impact. On the other hand, if the distance of the two mutations is long, then these mutations will likely not have common functional impact. Based on the hypothesis and the allele frequency values, we proposed the following equation to assign a score to each mutation:

$$
A c c_{\text {score }}^{(i)}=\frac{1}{n} \sum_{k=1}^{n} A F_{k, i}+\frac{1}{\log \left(d_{\min }(i)+1\right)+1},
$$

where $A c c_{\text {score }}^{(i)}$ is the accumulation score of mutation $i$, $n$ is the number of samples in a population and $d_{\min }(i)$ is the minimum distance of mutation $i$ with its vicinity mutations (mutations $i+1$ and $i-1$ ):

$$
d_{\min }(i)=\min \left[d_{(i, i-1)}, d_{(i, i+1)}\right],
$$

where $d_{(i, j)}$ is the distance of mutations $i$ and $j$ in base pairs (bp). Finally, to obtain the accumulation score of a gene, we selected the maximum accumulation score across its mutations:

$$
A c c_{\text {score }}^{[g]}=i=1 \ldots \operatorname{mmax} A c c_{\text {score }}^{(i)}
$$


where $m$ is the number of mutations occurred in gene $g$. The accumulation score of a gene represented its significance in a population.

\section{Pathogenicity score of mutated genes}

In this approach, unlike the mutation accumulation method, each sample was examined individually. A variety of mutation interpreters and databases have been designed to help understand the functional significance of genetic variants concerning their potential impact on genes and cancers. These tools can distinguish pathogenic mutations from benign ones using protein sequence, biochemical characteristics, and evolutionary information. Each mutation interpreter leveraged different methods and resources to predict the pathogenicity of a mutation. For this reason, a mutation interpreter might identify a mutation as benign, while another interpreter might detect it as a pathogenic mutation. In this study, we gathered the results of four different mutation interpreters (see Supplementary Methods). Our method prioritized pathogenic mutations rather than benign ones. For instance, in Supplementary Figure S1B, the first mutation was reported as benign (-7.8), benign (-6.2), pathogenic (6.1), and pathogenic (5) by InterVar, ClinVar, SIFT, and CADD, respectively. Next, we selected the highest score (most pathogenic) as the final mutation score (e.g., for the first mutation, 6.1 is the highest score). Finally, for calculating a gene score, we summed up all mutation scores in which their pathogenicity scores were higher than 0 (pathogenic). The use of a consensus method for assigning pathogenicity score to each gene was modified from the CoLaSp model [21].

\section{Gene microarray analysis}

The study cohort was composed of two cases of DLBCL in effusions (40 y/o male with GCB-type DLBCL in ascites and $55 \mathrm{y} / \mathrm{o}$ male with ABC-type DLBCL in pleural effusion) and two cases of DLBCL in solid organs (34 y/o female with $\mathrm{ABC}$-type DLBCL in anterior mediastinum and 36 y/o female with GCB-type DLBCL in mediastinal lymph node). Total RNA was extracted by Trizol Reagent (Invitrogen, USA) according to the instruction manual. Purified RNA was quantified at OD260 $\mathrm{nm}$ by using a ND-1000 spectrophotometer (Nanodrop Technology, USA) and qualified by using a Bioanalyzer 2100 (Agilent Technology, USA) with RNA 6000 nano labchip kit (Agilent Technologies, USA). Agilent microarray hybridization chamber kits were used for experiments (G2534A, Agilent Technologies, Palo Alto, CA, USA). Total RNA from DLBCL cells was used to prepare biotinylated RNA according to the manufacturer's recommendation. Ratios for GAPDH and $\beta$-actin $\left(3^{\prime} / 5^{\prime}\right)$ were within acceptable limits. After RNA isolation, two aliquots of $0.2 \mu \mathrm{g}$ of RNA were linearly amplified and fluorescently labeled with either Cy3-CTP (DLBCL in effusions) or Cy5-CTP (DLBCL in lymph nodes) with the Agilent Low Input Quick Amp Labeling Kit (Agilent Technologies). Equal amounts $(0.3 \mu \mathrm{g})$ of cyaninelabeled samples were hybridized to Agilent $8 \times 60 \mathrm{~K}$ Microarray chip (Agilent Technologies) according to the manufacturer. The microarray was scanned using an Agilent Microarray Scanner, and the scan was quantified using Agilent Feature Extraction software (version 10.5.1.1) and normalized using Rank consistency linear LOWESS with minimum background correction. Differentially expressed gene sets were identified using significance analysis of microarrays (SAM) and only those with positive or negative changes of 2.0 -fold or more were included. Hierarchic clustering was performed.

\section{Bioinformatics and pathway analysis}

Gene Set Enrichment Analysis (GSEA, UC San Diego, CA, USA) was used to determine the enriched genes from the cDNA microarray of DLBCL in effusions vs. DLBCL in solid organs. An enriched score was generated for each priori defined gene set based on the number of enriched genes. The settings and parameters are described in Table 2. According to an enriched score, we identified the gene sets that were positively and negatively correlated with the input data. Ingenuity Pathway Analysis software (IPA, Qiagen, Redwood City, CA, USA) was used to perform pathway analysis by subjecting selected genes. Known functional networks were tested for enrichment based on canonical pathways, relationship to upstream regulators, molecular and cellular functional groups, and associated network functions.

Table 2 The parameters and the settings in Gene Set Enrich Analysis (GSEA)

\begin{tabular}{ll}
\hline Chip Platform & $\begin{array}{l}\text { Human_Gene_Symbol_with_- } \\
\text { Remapping_MSigDB.v7.4.chip }\end{array}$ \\
\hline Gene sets database & C2.cp.kegg.v7.4.symbols.gmt [Curated] \\
Number of permutations & 1000 \\
Permutation type & gene_set \\
Enrichment statistics & Weighted \\
Metric for ranking genes & Signal2Noise \\
$\begin{array}{ll}\text { Min size (exclude smaller } \\
\text { sets) }\end{array}$ & 15 \\
$\begin{array}{l}\text { Max size (exclude larger } \\
\text { sets) }\end{array}$ & 500 \\
$\begin{array}{l}\text { Collapsing mode for probe } \\
\text { sets } \geq 1 \text { gene }\end{array}$ & Max_probe \\
Normalize mode & meandiv \\
\hline
\end{tabular}


DLBCL cell lines and EBV-transformed lymphoblastoid cell lines ( $L C L)$

To validate the clinical significance of genes yielded from the above bioinformatic analyses, quantitative realtime PCR, Western blotting, and immunohistochemical analysis on clinical samples were performed. The DLBCL cell lines and LCL (Supplementary Table S1) were cultured at $37{ }^{\circ} \mathrm{C}$ and $7 \% \mathrm{CO}_{2}$ in RPMI 1640 medium (Gibco/BRL, Grand Island, NY, USA) supplemented with $10 \%$ heat-inactivated fetal bovine serum (FBS), $4 \mathrm{mM}$ of glutamine, $75 \mathrm{units} / \mathrm{ml}$ of streptomycin, and 100 units $/ \mathrm{ml}$ of penicillin. Cell viability was determined using the trypan blue exclusion test or MTT (3[4,5-dimethylthiazol-2-yl]-2,5-diphenyltetrazolium bromide) assay.

\section{TaqMan quantitative real time PCR (TaqMan qRCR)}

Total RNA of B-cell lymphomas and cells from pleural fluid of lymphoma patients were extracted by QIAzol (\#79,306, QIAGEN) according to the manufacturer's instruction. One microgram of total RNA was reverse transcribed into cDNA using High-Capacity cDNA Reverse Transcription Kit (\#4,368,814, Thermo). Gene expression was determined by TaqMan quantitative realtime polymerase chain reaction (qPCR) using ChamGE Probe qPCR Master Mix (CGE-03, TopGen Biotech). Sequences of PCR primers and TaqMan probes are listed as follows:

\begin{tabular}{|c|c|c|}
\hline TP53BP2 & Probe & ACAAACTTGCGTAAAACTGGCTC \\
\hline & Forward & AAGACTCGGTGAGCATGCG \\
\hline & Reverse & CCTCATTCCATGAGCGATACG \\
\hline \multirow[t]{3}{*}{ SLC35G6 } & Probe & AGGAAAGATGGCTGGCAGTC \\
\hline & Forward & CACTCCAACCATGTCACAATGG \\
\hline & Reverse & GTCAGGCGGGTTCAAGTAGG \\
\hline \multirow[t]{3}{*}{ PDIA4 } & Probe & ACTGAAGCCAGTCATCAAATCCC \\
\hline & Forward & CATGGAGCCAGAGGAGTTTGAC \\
\hline & Reverse & GACGGGTCCCTTGTTGTTCTT \\
\hline \multirow[t]{3}{*}{ MUC4 } & Probe & AGCTCTTTGAGAATGGGACGTTG \\
\hline & Forward & CAATGCTGAGGATGCCAACTT \\
\hline & Reverse & TGCTAGAATCTCCAGAGTGAATGG \\
\hline \multirow[t]{3}{*}{ MDM2 } & Probe & AGAATTGGCTTCCTGAAGATAAAGGG \\
\hline & Forward & CACTTCATGCAATGAAATGAATCC \\
\hline & Reverse & TGAGTTTCCAGTTTGGCTTTCT \\
\hline \multirow[t]{3}{*}{ IL13RA1 } & Probe & ACTTCCCGTGTGAAACCTGATC \\
\hline & Forward & AATAATGGTCAAGGATAATGCAGGA \\
\hline & Reverse & CATCATTGTGGAAGGAGAGGTTT \\
\hline \multirow[t]{2}{*}{ HDAC1 } & Probe & ATGGAAATCTATCGCCCTCACAA \\
\hline & Forward & CTCACCGAATCCGCATGAC \\
\hline
\end{tabular}

TaqMan quantitative real time PCR (TaqMan qRCR) (Continued)

\begin{tabular}{lll}
\hline TP53BP2 & Probe & ACAAACTTGCGTAAAACTGGCTC \\
\hline \multirow{2}{*}{ ARAP3 } & Reverse & GCTGTGGTACTTGGTCATCTCCT \\
& Probe & ACTTACAGCGGCTTCCTGTACT \\
& Forward & CTGGCCTCTGCCCTCAGA \\
& Reverse & GAGGGTCCAGCTTGTTGCT \\
ACTB & Probe & AGGCACCAGGGCGTG \\
& Forward & ATGTGCAAGGCCGGCTT \\
& Reverse & CTCTTGCTCTGGGCCTCGT \\
\hline
\end{tabular}

\section{Western blot analysis}

Total lysates of B-cell lymphomas and cells from pleural fluid of lymphoma patients were extracted by RIPA lysis buffer $(50 \mathrm{mM}$ Tris- $\mathrm{HCl} / \mathrm{pH}$ 8.0, $150 \mathrm{mM} \mathrm{NaCl}, 0.5 \%$ sodium deoxycholate, $1 \%$ Nonidet P-40, $0.1 \%$ SDS, 1 $\mathrm{mM}$ DTT, $10 \mathrm{mM} \beta$-glycerol phosphate, and $1 \mathrm{mM}$ EGTA) supplemented with protease inhibitor cocktail (P8340, Sigma). Protein concentration is measured using Protein Assay Kit $(\# 5,000,006$, Bio-Rad). Anti-ERp72 (PDIA4) antibody (\#2798, Cell Signaling), anti-TP53BP2 antibody (ab181377, Abcam) and anti- $\alpha$-tubulin (T6199, Sigma,) were used for western blot analysis.

\section{Immunohistochemical analysis}

Immunohistochemical staining was performed on deparaffinized tissue sections of formalin-fixed material, pre-treated with the Epitope Retrieval Solution 2 (EDTA, $\mathrm{pH}$ 9.0). The procedures were performed using the Bond-Max Automated IHC stainer (Leica Biosystems Newcastle Ltd, Australia). The primary antibodies and working dilutions were as follows: MUC4 (1:100, 8G7, mouse monoclonal, Zeta Corporation, Taichung, Taiwan), MDM2 (1:100, IF2, mouse monoclonal, Invitrogen, Thermo Fisher Scientific, Waltham, MA USA), and HDAC1 (1:400, 4E1, mouse monoclonal, GeneTex, Hsinchu, Taiwan). Appropriate positive and negative controls were used. Counterstaining was carried out with hematoxylin, and images were photographed using a digital microscope camera (DP12; Olympus Co., Tokyo, Japan) and processed by Adobe Photoshop version 8.0 software (Adobe Systems Incorporated, San Jose, CA, USA). The staining was deemed positive when tumor cells showed nuclear expression of MDM2 in $\geq$ $10 \%$ [22] and HDAC1 in $\geq 50 \%$ [23] of the tumor cells, as described previously. For MUC4, cytoplasmic staining in $\geq 10 \%$ of the tumor cells was graded as positive. The independent cohort of additional 51 DLBCL cases with $(n=31)$ or without $(n=20)$ lymphomatous effusions are listed in Supplementary Table S2 [4]. 


\section{Statistical analysis}

Appropriate statistical tests were used to examine the relationships and correlations between variables, including $X^{2}$-test. Overall survival was measured from initial diagnosis to death from any cause, with follow-up data of surviving patients assessed at the last contact date. Estimates of overall survival distribution were calculated using the method of Kaplan and Meier. Timeto-event distribution was compared using the log-rank test. The analyses were carried out using SPSS statistical software (SPSS, Inc., Chicago, IL, USA).

\section{Results}

WES information of 9 effusion-associated DLBCL samples is provided in Supplementary Table S3. The mean depth of high-quality sequences on targets ranged from 197.4x to 377.2x, with median 301.9x. Because matched germline DNA was unavailable, somatic variants were identified by comparing with genetic variation databases. Variants present in public and in-house databases (gnomAD East Asian and Taiwan biobank) with a minor allele frequency (MAF) $>1 \%$ were filtered out. Supplementary Figure S2 shows the number of these potentially somatic variants in each sample. A total of 3,919 single nucleotide variants (SNVs, median per sample: 476, range: 403-555) and 209 insertions/deletions (indels, median per sample: 30, range: 18-37) showed potentially protein-changing features (Supplementary Table S4 Excel file). WES data are available in the National Center for Biotechnology Information (https:// submit.ncbi.nlm.nih.gov/), BioProject: PRJNA740363 (https://dataview.ncbi.nlm.nih.gov/object/PRJNA740363 ?reviewer $=49$ scseu631s44tsbpgt64h0pmi).

\section{Mutation scoring method distinguishes higher genetic alterations in migration/invasion-associated pathways in effusion-associated DLBCL}

The routes of lymphoma cells into body cavities might spread via blood or lymphatic vessels [24]. The serous fluids in body cavities circulate from parietal vessels to visceral vessels. Hence, the existence of lymphoma cells in body fluids highlights the increased capability of the lymphoma cells to spread. Furthermore, this capability might be a manifestation of a more aggressive metastatic dissemination repertoire, such as migration, invasion, and adhesion. For this reason, we extracted migration/ invasion regulator genes in various pathways. The extracted genes belonged to the one of the following pathways or groups: B cell receptor (BCR), NFKB, tolllike receptors (TLR), focal adhesion kinase (FAK), BCL10-CARD11-MALT1 (BCM) complex, cytokines, somatic hypermutation, leukocyte transendothelial migration, glycoproteins, and transmembrane proteins (Supplementary Table S5 Excel file).
Next, we compared the accumulation score of the migration/invasion regulator genes in the above pathways and groups between the two DLBCL cohorts (with versus without lymphomatous effusion). As shown in Fig. 1, the boxplots compare the accumulation scores of the two cohorts for different pathways and groups. Interestingly, the genes in boxplots for the effusionassociated DLBCL cases showed significant higher accumulation scores compared to those of non-effusionassociated DLBCL cases. The mean score in the effusion-associated DLBCL dataset was higher than 0.4. By contrast, the mean value of the accumulation score in the non-effusion DLBCL dataset was less than 0.2 in most cases. The Supplementary Table S6 Excel file contains the accumulation scores of all genes in both datasets.

\section{Identification of the pathogenic and mutated genes in effusion-based DLBCL samples}

Mutations and SNVs are rich sources for detecting the biomarkers of various diseases. Here, we considered mutations/SNVs of the samples with tumors in effusions. The details of the mutations/SNVs of the effusion-associated DLBCL dataset are listed in Supplementary Figures S3-S6. We obtained the genes affected by SNVs, indels, or both in at least five samples (Fig. $2 \mathrm{~A}$ ). Supplementary Figure S7 shows the stacked bar charts of SNVs and indels in the effusion-associated and nodalbased DLBCL cohorts, and Supplementary Figure S8 comprises all genes affected by splice, indels, missense, multiple hits, stop-loss, and stop-gain mutations in at least one effusion-associated DLBCL sample. Although Fig. $2 \mathrm{~A}$ and Supplementary Figure S8 contain helpful information about the genes with their characteristics, there is no connection between the number of mutations in a gene and the malignant potential of an abnormal gene. For this reason, we extracted the genes that were detected as pathogenic in at least five effusion-associated DLBCL samples (Fig. 2B). As seen in Fig. 2, MUC4 (pathogenic in 9/9 samples), SLC35G6 (8/9), ARAP3 (7/ 9), SLC9B1 (6/9), DDX11 (6/9), MUC16 (5/9), and HNRNPC (5/9) are reported in both pathogenic and the mutated genes lists. Surprisingly, all genes that are reported in the two figures are mutated or pathogenic in a small number of the non-effusion-associated DLBCL samples except for $M U C 4$, which was also frequently represented in non-effusion-associated DLBCL and warranted further validation.

\section{Gene set enrichment and pathway analyses}

To decipher the gene expression profiling (GEP) responsible for lymphoma seeding in effusions, we 


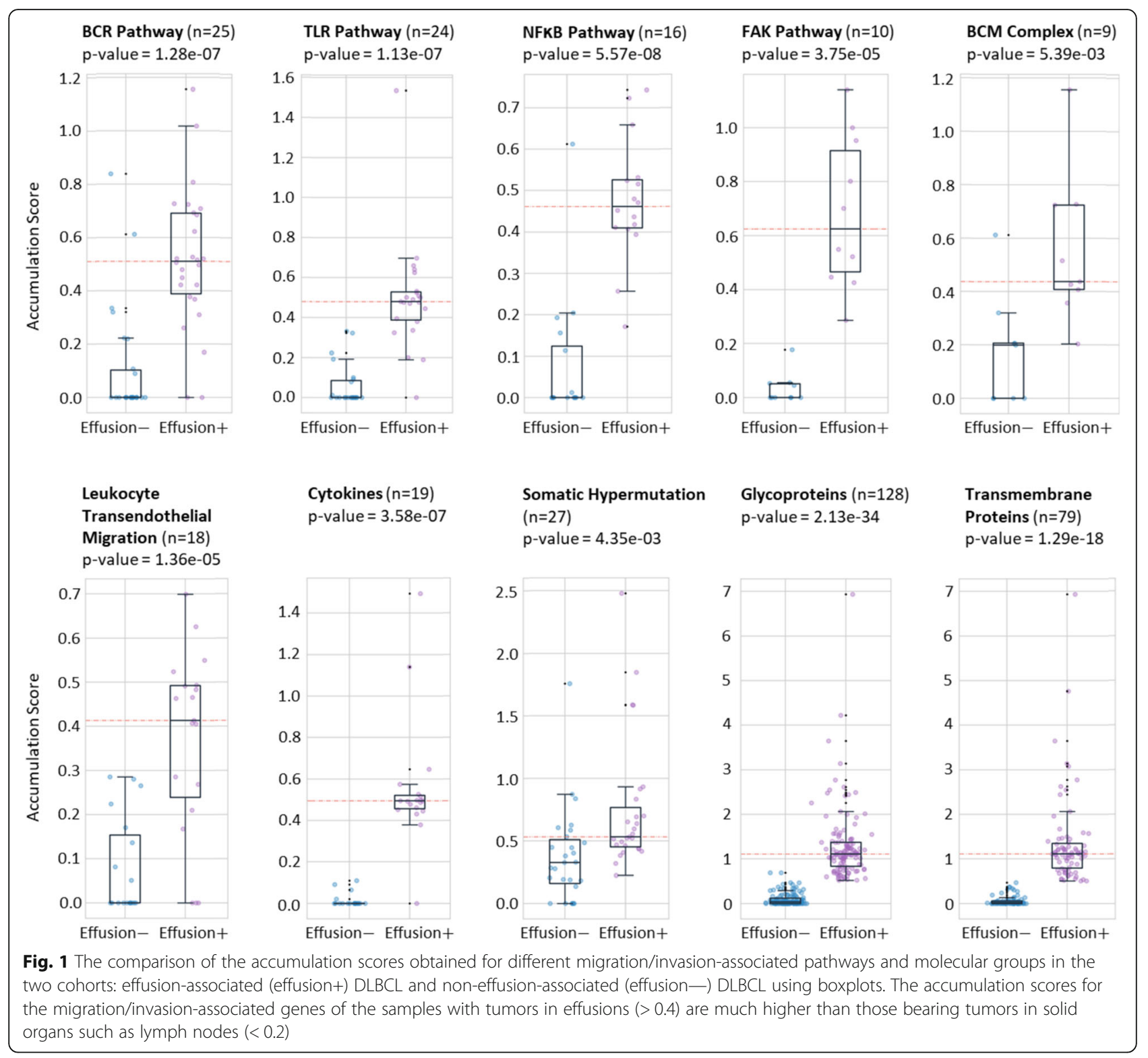

additionally used cDNA microarrays to search for differentially expressed genes between effusionassociated DLBCL cases versus DLBCL without effusion. Since both groups of DLBCL cells were from the sites with different microenvironments, that is, one was embedded in solid matrix and the other floated in effusions, we carefully excluded genes engaged in microenvironment and matrix remodeling to minimalize the bias. As shown in Supplementary Table S7 Excel file, the genes with 1.5-fold changes of expression intensity in the effusion group were consequently subjected to the statistical simulations and analysis using Ingenuity Pathway Analysis (IPA) and Gene Set Enrichment Analysis (GSEA) software to identify canonical pathways.
GSEA analysis showed that chronic myeloid leukemia (enrichment score $(E S)=0.55$; p-value $=0.010$ ), cell cycle $(\mathrm{ES}=0.53 ; \mathrm{p}$-value $=0.000)$, MAPK $(\mathrm{ES}=0.45 ; \mathrm{p}$-value $=$ 0.007), WNT $(E S=0.57 ; p$-value $=0.006), \mathrm{TGF} \beta(E S=$ 0.56; $\mathrm{p}$-value $=0.014)$, VEGF $(E S=0.80 ; \mathrm{p}$-value $=0.000)$, TLR (ES $=0.61 ; \mathrm{p}$-value $=0.004)$, and JAK-STAT $(\mathrm{ES}=$ $0.50 ; \mathrm{p}$-value $=0.044)$ pathways were significantly activated in samples of DLBCL associated with lymphomatous effusions (Fig. 3 and Supplementary Figures S9). In addition, the single-sample analysis (ssGSEA) and Gene Set Variation Analysis (GSVA) calculating sample-wise gene set enrichment showed results consistent with GSEA findings (Table 3). On the other hand, IPA revealed that samples bearing tumor in effusions were 


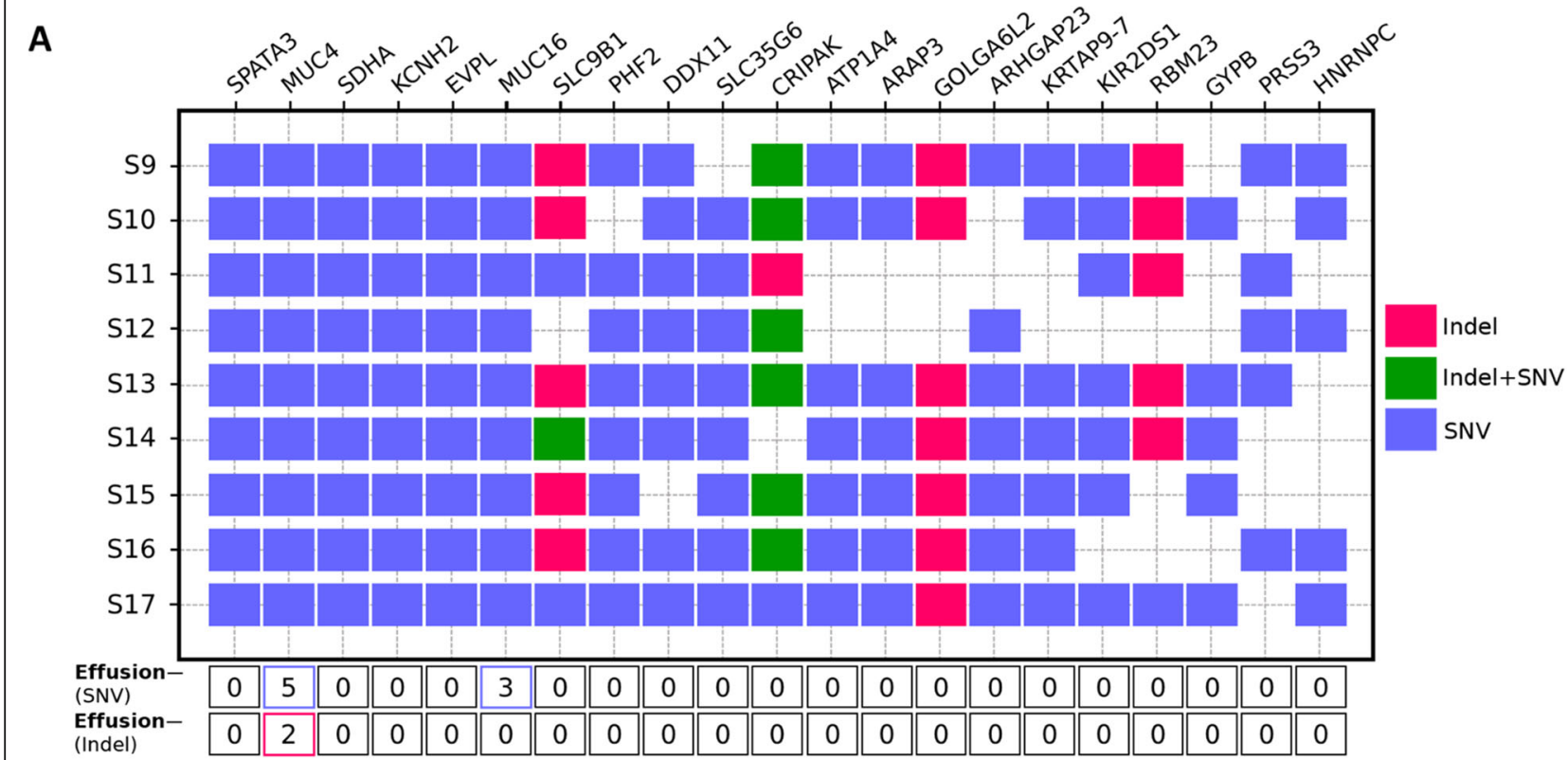

B

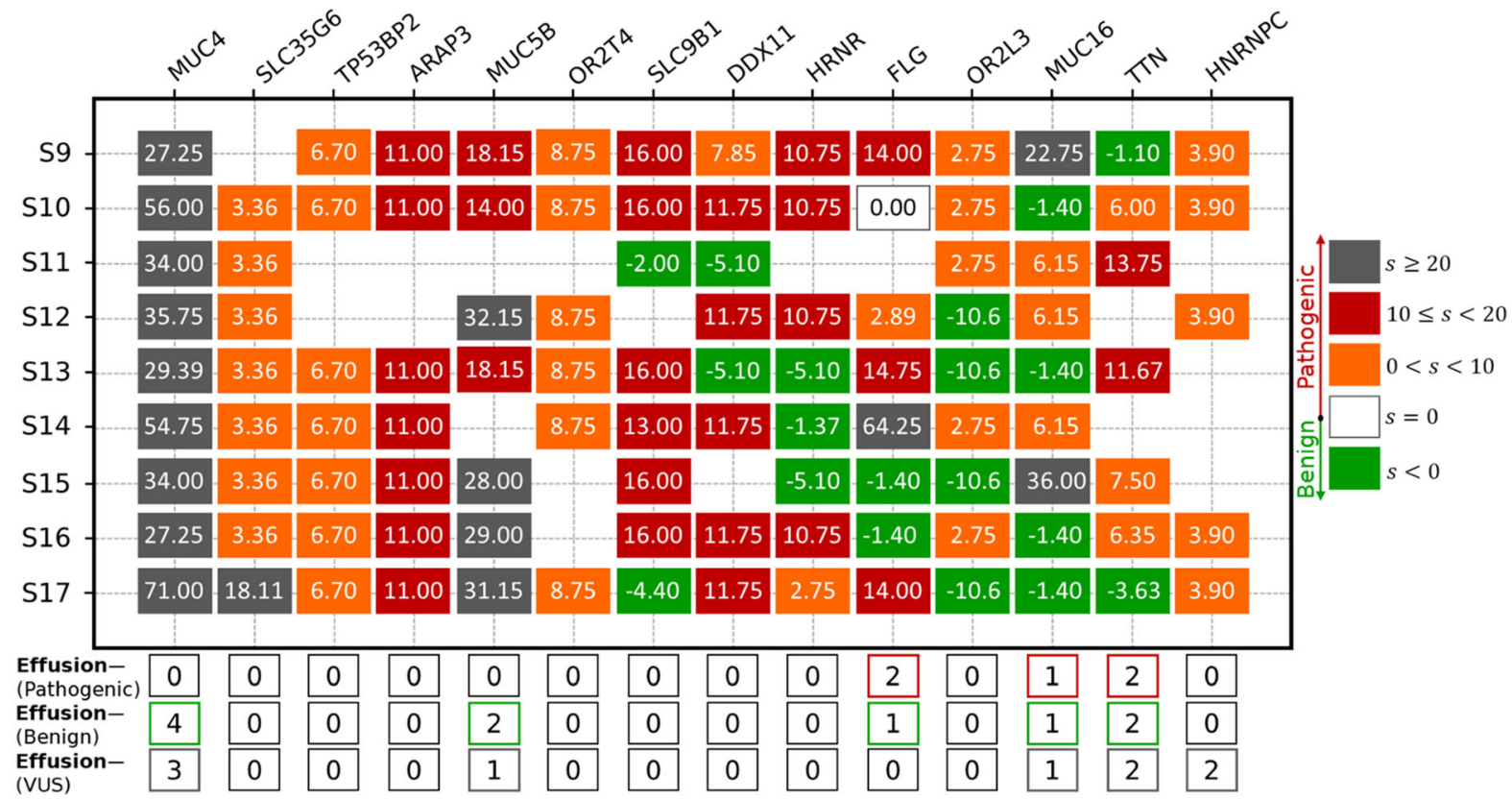

Fig. 2 Genes affected by mutations in at least five effusion-associated (effusion+) DLBCL samples. (A) The samples containing at least one insertion/deletion (Indel) mutation, SNV, or both indel/SNV are represented by pink, blue, and green squares, respectively. The bottom of the figure shows the number of non-effusion-associated (effusion-) samples that contain SNVs and Indels. (B) Genes that have pathogenic mutation(s) in at least five effusion-associated (effusion+) DLBCL samples. Genes containing benign mutation(s) are shown by green ( $s<0)$. Genes that have no pathogenic mutation and at least one variant of uncertain significance (VUS) are shown by white $(s=0)$. Genes that consist of at least one pathogenic mutation are shown by orange $(0<s<10)$, red $(10<=s<=20)$, or gray $(s>20)$ depending on the number of pathogenic mutations. The more positive score is interpreted as more pathogenic

significantly associated with pathways involving p53 signaling, nucleotide excision repair (NER), checkpoint kinase $(\mathrm{CHK})$ proteins in cell cycle, DNA replication, sumoylation (SUMO), and p38 MAPK pathways. The details of each pathway extracted by IPA are shown in
Supplementary Table S8 Excel file. We extracted the partner proteins involved in the pathways identified by IPA and GSEA. Figure $4 \mathrm{~A}$ illustrates the mutation accumulation scores of these proteins, which were also present in the NGS dataset. In this figure, we excluded 


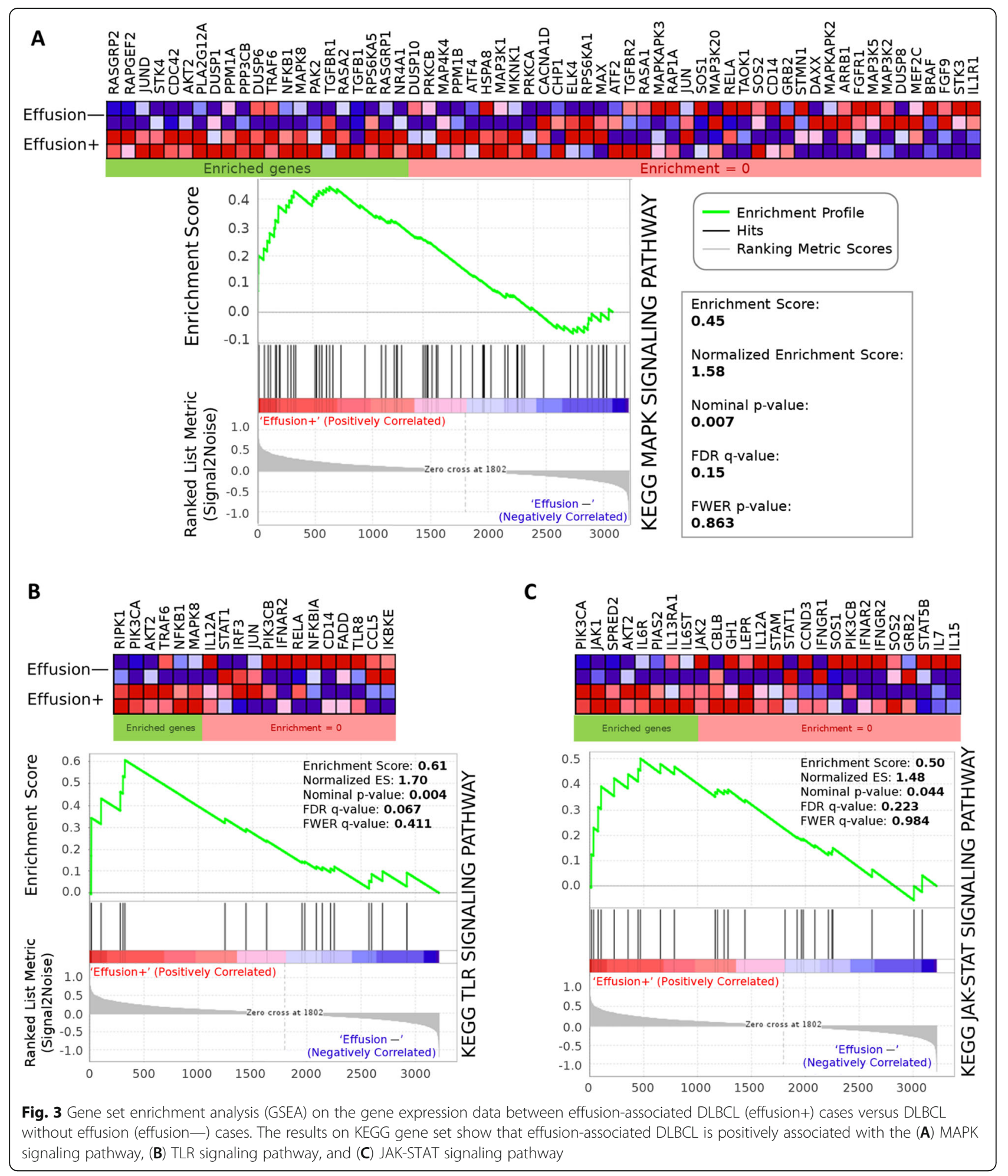

the proteins in which their scores in both effusionassociated and non-effusion DLBCL groups were zero. The proteins with a higher score in the effusionassociated cohort compared to the non-effusionassociated cohort were represented by the purple color.
In contrast, the blue-colored proteins had a higher score in the non-effusion-associated DLBCL cohort. As seen in Fig. $4 \mathrm{~A}$, most of the genes identified by GSEA and IPA had higher accumulation scores in the effusionassociated DLBCL cohort, that is, the genes in Fig. 4 A 
Table 3 Results of Gene Set Variation Analysis (GSVA) and single-sample GSEA (sSGSEA)

\begin{tabular}{lllllllll}
\hline & GSVA & & \multicolumn{5}{c}{ ssGSEA } \\
\hline Signaling pathway & $\mathrm{E}(+) 1$ & $\mathrm{E}(+) 2$ & $\mathrm{E}(-) 1$ & $\mathrm{E}(-) 2$ & $\mathrm{E}(+) 1$ & $\mathrm{E}(+) 2$ & $\mathrm{E}(-) 1$ & $\mathrm{E}(-) 2$ \\
MAPK & 0.158 & 0.312 & -0.236 & -0.244 & 0.175 & 0.170 & 0.061 & 0.059 \\
Toll-like receptor & 0.160 & 0.168 & -0.142 & -0.177 & 0.170 & 0.177 & 0.113 & 0.116 \\
JAK/STAT & 0.359 & 0.208 & -0.014 & -0.498 & 0.083 & 0.076 & -0.043 & -0.059 \\
\hline
\end{tabular}

Both GSVA and ssGSEA were implemented using "GSVA" package in $\mathrm{R}$

were present in both microarray and NGS datasets and showed higher mutation scores in effusion-associated DLBCL cohort. In Fig. 4B, we highlighted the six pathways most associated with highest/lowest $\mathrm{z}$-score values. The positive and negative $\mathrm{z}$-score values indicate the activation and the inhibition of pathways, respectively, in the effusion-associated DLBCL cohort. Accordingly, we found activation of SUMOylation and p38 MAPK pathways (Supplementary Figures S10-S11) as well as inhibition/downregulation in cell cycle control of DAN replication, NER, cell cycle checkpoint control, and p53 signaling pathways (Supplementary Figures S12-S15).

Validation of mutations in selected genes in cell lines and clinical samples of DLBCL

We validated the expression of relevant genes yielded by NGS (Fig. 2B) and GEP (Fig. 4B) analyses in different cell lines and clinical samples of DLBCL. As shown in
Fig. 5 A, quantitative reverse transcription PCR (qRTPCR) confirmed the higher expression of SLC35G6, MUC4, TP53BP2, PDIA4, HDAC1 and MDM2 in most DLBCL cell lines compared with LCL (lymphoblastoid cell lines). The mRNA levels of $H D A C 1, M D M 2$, and PDIA4 were highly expressed in most clinical samples in comparison with $\beta$-actin (Fig. 5B). Western blot (WB) analysis also confirmed that the protein levels of PDIA4 and TP53BP2 were highly expressed in most DLBCL cell lines (Fig. $6 \mathrm{~A}$ ) and clinical samples (Fig. 6B). The lack of available SLC35G6 antibody prevented the validation of protein expression for this marker (https://www. antibodypedia.com/gene/82013/SLC35G6).

An independent cohort of 51 DLBCL cases (Supplementary Table S2) with $(\mathrm{n}=31)$ and without $(\mathrm{n}=$ 20) lymphomatous effusions were immunohistochemically studied to validate the role of MUM4, HDAC1 and MDM2 overexpression in the formation of lymphomatous effusions. We found that overexpression of HDAC1

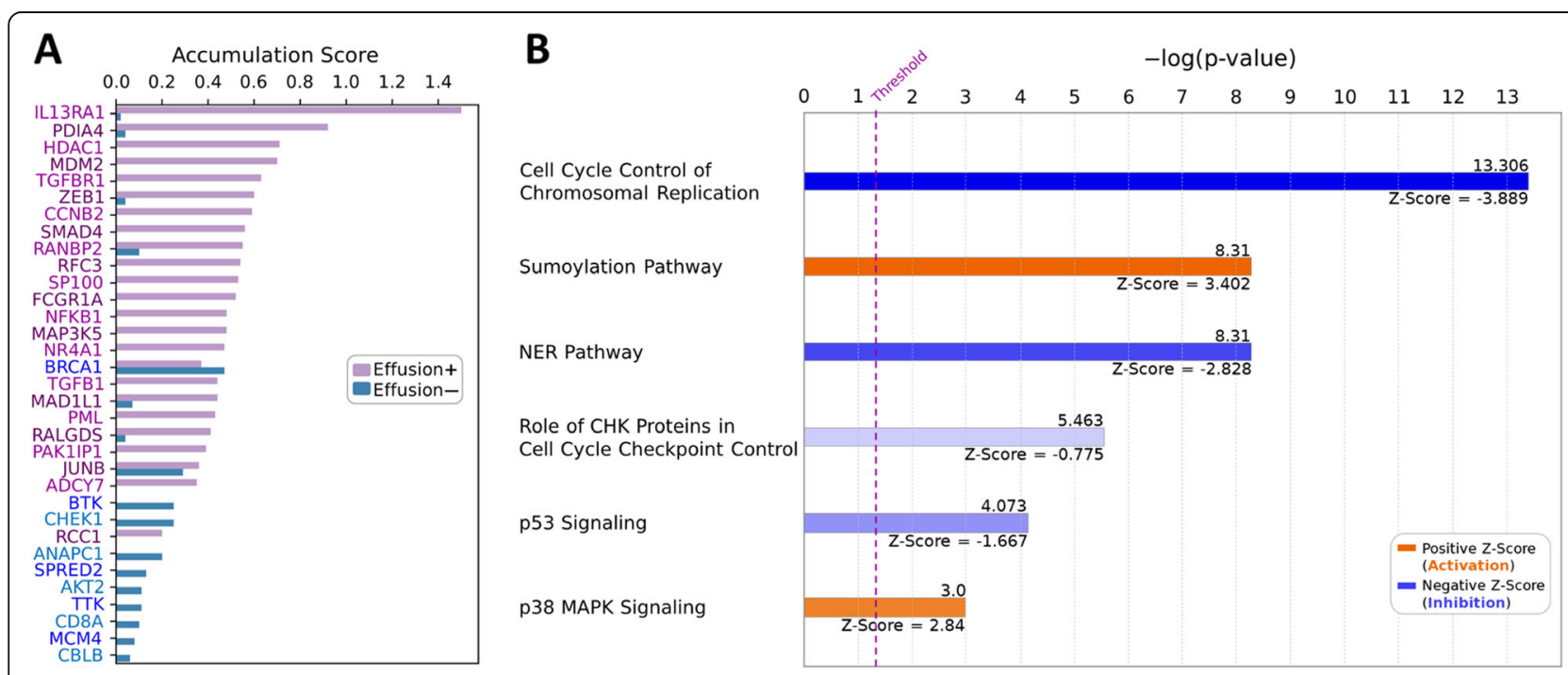

Fig. 4 The accumulation scores of the partner proteins involve in the different pathways identified by IPA and GSEA. (A) The proteins with a high score in the effusion-associated (effusion+) cohort and non-effusion-associated (effusion-) cohort are represented by purple and blue, respectively. (B) The pathways that are positively and negatively associated with effusion-associated DLBCL detected by IPA. We highlighted the six maximum and minimum $z$-score values. The orange bars (positive $z$-score) indicate the activation of pathways, and the blue bars (negative $z$ score) indicate the inhibition of pathways 


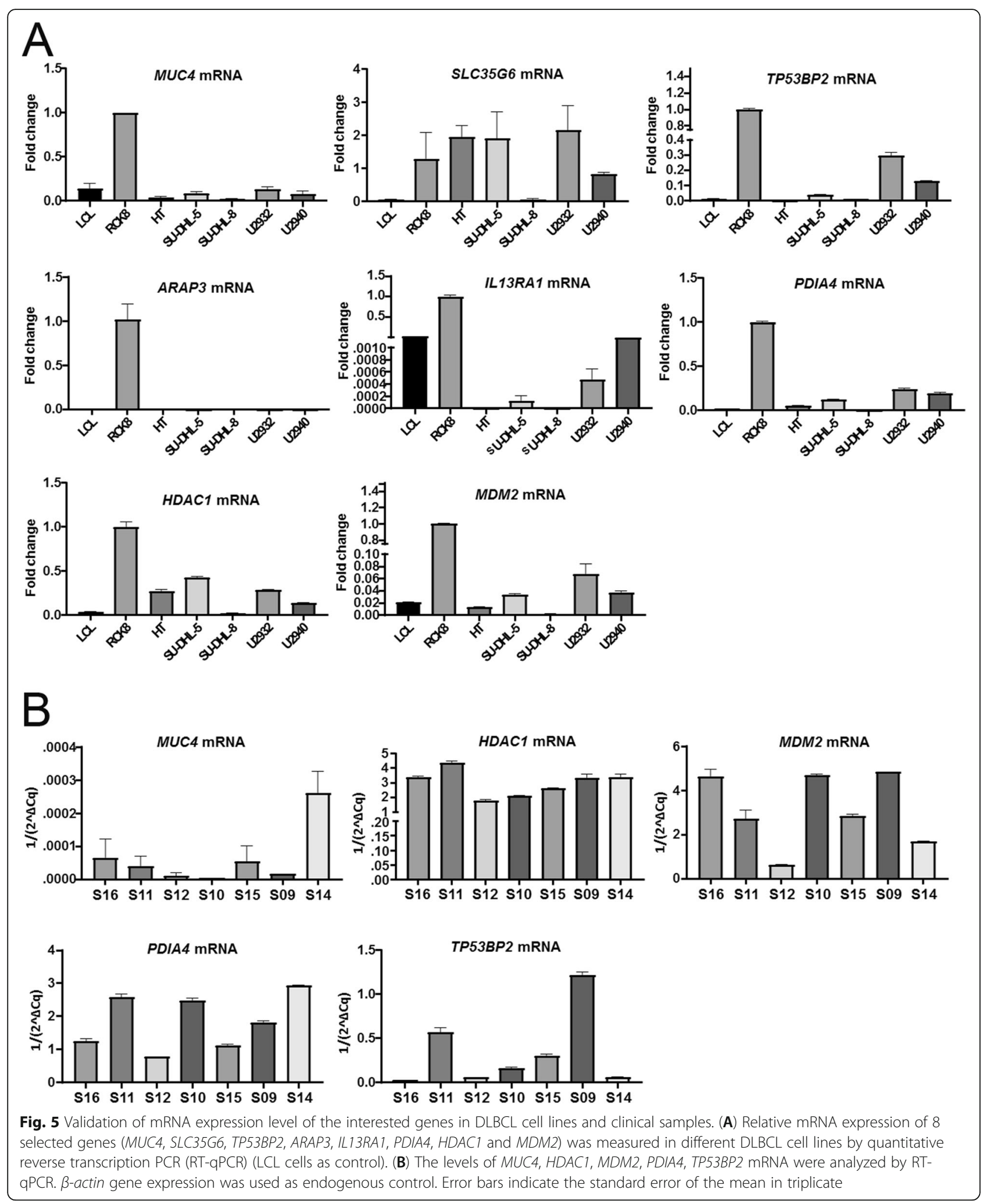

(Fig. $6 \mathrm{C}$ ) and MDM2 (Fig. 6D) correlated with the presence of lymphomatous effusions (Fig. 6E), and HDAC1 overexpression also was associated with a poorer prognosis $(\mathrm{p}=0.005$, Fig. $6 \mathrm{~F})$. The expression of HDAC1 and MDM2 in non-effusion DLBCL cases and survival analyses for other clinicopathologic factors are shown in 


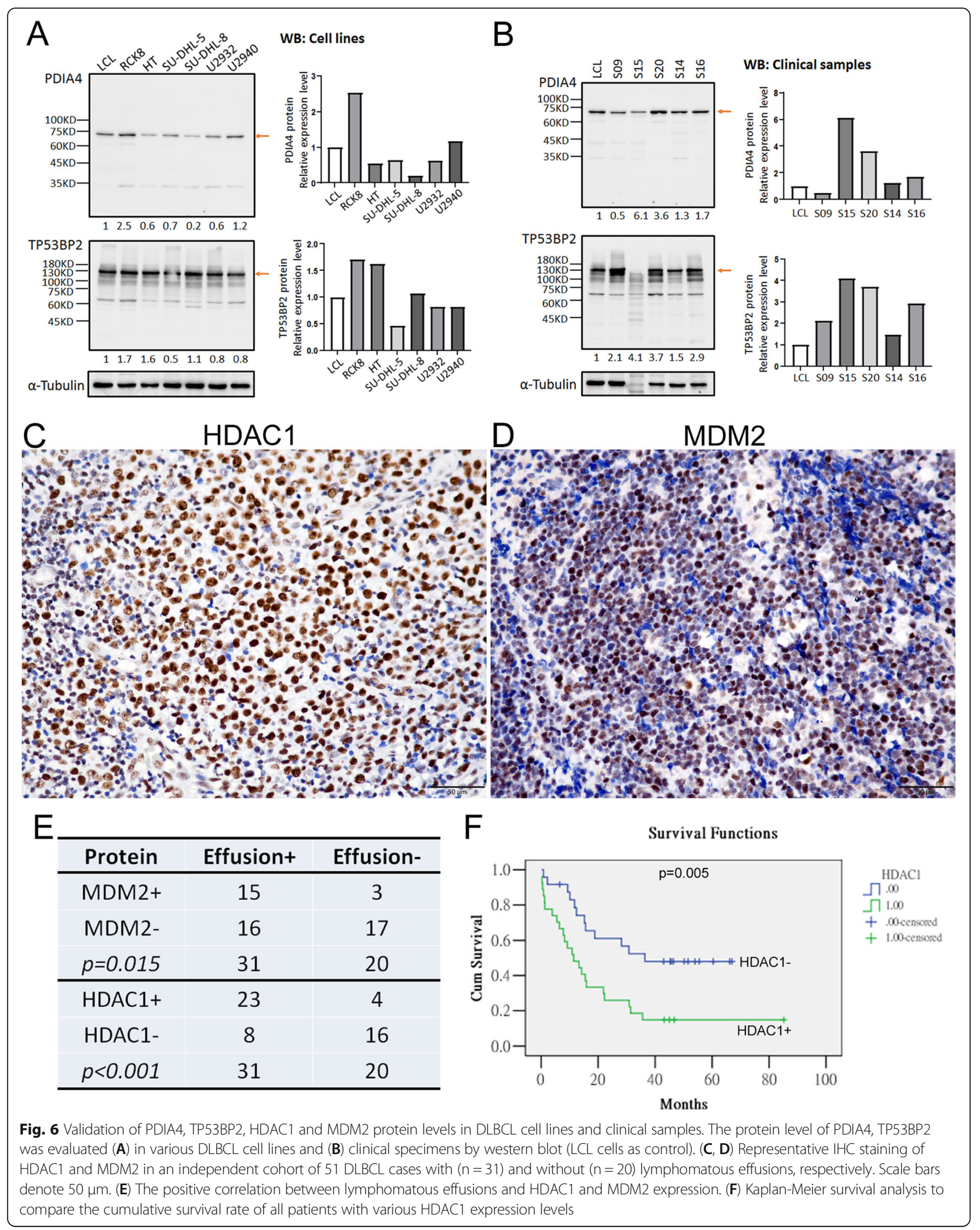


Supplementary Figure S16. Notably, although MUC4 was found to be pathogenic in the NGS analysis, all 51 DLBCL cases were negative for MUC4 expression.

\section{Discussion}

In this study, we designed a method that assigned an RMD-based score to each gene across a population. We selected the genes that had high score in the cases of DLBCL associated with lymphomatous effusions and a low score $(\approx 0)$ in the nodal-based DLBCL cases without effusions. Furthermore, we utilized four different mutation interpreters to identify pathogenic genes. The genes that were reported as pathogenic in most effusionassociated DLBCL samples but were benign in the nodal-based DLBCL samples without effusions might be associated with effusion-based DLBCL. Interestingly, we found that the genes with high RMD-based or pathogenicity scores were related to tumor invasion, migration, and adhesion. Finally, we validated the role of TP53related and chromatin remodeling pathways in pathogenesis of DLBCL lymphomatous effusions in an independent cohort. We found that DLBCL-associated lymphomatous effusions may be associated mechanistically with TP53-MDM2 pathway and HDAC-related chromatin remodeling mechanisms.

It is also interesting that the effusion cases harbor much more Indels and SNVs than the non-effusion cases (Supplementary Figure S7). These findings are not wellknown. The non-effusion group from TCGA was nodalbased and analyzed by using Mutect2 to call somatic variants in tumor and matched normal samples. Variants with MAF $>1 \%$ in public and in-house databases (gnomAD East Asian and Taiwan biobank) were excluded (filtered) for further analysis. In contrast, for the effusion-positive group, we used Freebayes to call potentially somatic variants in tumor samples, considered only exonic or splicing variants, and removed synonymous SNV. Thus, the possible causes for that the effusion cases harbor much more Indels and SNVs than the noneffusion cases are described as follows: first, the filter strategy: TCGA group first used matched normal tissues to subtract potential candidate genes but we did not; second, the tumor cell biology: spreading (metastatic) tumor cells acquire additional mutated genes to accomplish the dissemination process than primary (lymph node) tumor cells. So, it is reasonable that the effusion cohort (metastatic tumors) carries much more mutated genes than the non-effusion cohort (primary tumors).

Focusing on the connection with migration/invasion pathways is important. Thus, we utilized various migration/invasion-associated pathways to calculate the mutation accumulation scores of their regulator genes in DLBCL with or without lymphomatous effusions. We found the following pathways more highly activated in the effusion-associated DLBCL cohort, including BCR, NFאB, TLR, FAK, BCM complex, cytokines, and glycoproteins. Extracellular stimuli activate and phosphorylate the MEKK1-MKK4 (or MKK7)-JNK-FAK signaling through FAK (PTK2); next, activated JNK phosphorylates Jun, Paxillin, or Spir [25], and phosphorylated Jun promotes cell migration. Phosphorylation of Paxillin accelerates turnover of cell adhesion, and promotes rapid cell motility [25]. The phosphorylated Spir also affects actin dynamics and cell migration. Furthermore, FGF and EGF growth factors activate FAK via the Ras-RafMEK-Erk-FAK signaling module [25].

One of the major regulators of lymphocyte survival, proliferation, and activation is the transcription factor $\mathrm{NF \kappa B}$ that provides association between chronic inflammation and lymphomagenesis. NFKB activation increases the production of anti-apoptotic factors and chemokines that trigger the migration of immune cells to inflammatory foci. These properties promote tumor cell survival and metastasis, while inhibit apoptosis [26]. The BCL10-CARD11-MALT1 (BCM) complex plays a key role in forming an essential connection between $\mathrm{NF} K \mathrm{~B}$ activation and the triggering of cell surface antigen receptors. Genetic and biochemical methods show that the connection between the BCM complex and activated $N F \kappa B$ functionally leads to migration and invasion [27]. The pathways that are involved in activating NFKB signaling can promote tumor growth. The interaction of pathogens with Toll-like receptors (TLRs) on the cell surface is known to activate the NFkB pathway. The activation of $N F \kappa B$ results in upregulation of interleukin (IL)-10, IL-16, IL-2, IL-1, and increased production of various pro-inflammatory cytokines [28]. The activation of TLR signaling in tumor cells stimulates metastasis and enhances the proliferation of cancer cells via angiogenic factors, such as MMP, VEGF, and IL-8 [29]. Inflammatory mediators such as cytokines can suppress the DNA mismatch repair system across various mechanisms, which subsequently result in genetic mutations [30].

Signaling through the BCR activates the cytoplasmic domain of integrin, causing a conformational alteration in the extracellular domain that induces cell migration [31]. The accumulation of somatic mutations in essential genes of the BCR pathway highlights the key role of $\mathrm{BCR}$ signaling in DLBCL tumorigenesis. Most $C D 79 A / B$ mutations result in deletions of large segments of the immunoreceptor tyrosine-based activation motif (ITAM) region [32]. These mutations have been shown to enhance BCR surface expression levels [32]. Glycosylation affects tumor growth and survival and promotes metastasis [33]. Aberrant glycosylation in tumors is linked with oncogenic transformation and plays a crucial role in progression, growth, and metastasis [34, 35]. Oliveira- 
Ferrer et al. [35] have shown that glycoproteins influence distinct stages of the metastatic process, such as migration and invasion of cancer cells.

We also highlighted the genes affected by mutations or detected as pathogenic in at least five effusionassociated DLBCL samples. Here, we discuss the connection between some of the genes with migration/invasion of cells. MUC4 facilitates metastases by promoting a group of tumor cells that diffuse into the bloodstream. A study has shown that MUC4 intensifies invasion and migration potential and promotes metastasis and oncogenesis [36]. MUC16 increases the invasion, migration, and proliferation of cancer cells in vitro, and also enhances tumorigenesis and metastasis in vivo [37]. ARAP3, which is found in the plasma membrane, is essential for lamellipodia formation after stimulation of the growth factor signaling on activation of PI3K pathway [38]. Wang et al. identified that downregulation of ARAP3 significantly inhibited the invasive and migratory abilities of thyroid cancer cell lines [39]. TP53BP2 (ASPP2) inhibition accelerates cell migration, invasion, and epithelial-mesenchymal transition in breast cancer cells [40]. Notably, although MUC4 was found to be pathogenic in the NGS analysis, all 51 DLBCL cases were negative for MUC4 expression. These data highlight the importance of validating protein expression on clinical samples. Alternatively, it is possible that MUC4 mutations lead to loss of function.

GSEA analysis revealed that a set of pathways such as WNT, VEGF, and JAK-STAT were significantly activated in samples of DLBCL-associated lymphomatous effusions. Aberrant activation of the WNT pathway promotes abnormal cellular behaviors such as cell motility, matrix invasion, and tumor progression [41]. Inhibition of VEGF signaling in colon cancer cell lines strongly inhibits cancer cell migration and invasion by regulatory proteins associated with cell motility [42]. JAK1 is the main activator of STAT3 in many cellular systems [43]. An earlier study has shown that the JAK-STAT signaling largely correlates with the invasion and migration [44]. Increased STAT3 expression has been reported in metastases at the leading edges of invasive cancers [45].

IPA analysis identified p53 signaling, nucleotide excision repair (NER), checkpoint kinase (CHK) proteins in cell cycle, DNA replication, sumoylation (SUMO), and p38 MAPK pathways that were significantly associated with samples bearing tumor in effusions. p53 is a tumor suppressor whose loss perturbs cell-cycle checkpoints. p53 inhibition also devastates pathways that reduce metastasis. p53 directly controls the transcription of genes that are involved in cell adhesion, motility, and invasion [46]. Dysregulation of p38 is associated with metastases and low survival rates [47]. Furthermore, a well-known anti-cancer drug, baicalein, inhibits cancer cell motility and metastasis through inhibition of the p38 signaling pathway [48]. The NER signaling pathway identifies and removes a wide variety of DNA damage. Aberrations in NER-associated genes have been illustrated in several malignancies with a potential impact on clinical issues [49]. The Ranbp2 protein relates to cancer cells, and genetic point mutations and translocations are associated with tumorigenesis [50]. Interaction of insulin-like growth factor-1 receptor (IGF1R) with Ranbp2 is essential for IGF1R sumoylation that plays a crucial role in cancer cell progression [51]. On the other hand, some polymorphisms of the SUMO-conjugating enzyme ubc 9 are associated with metastasis and invasion [52]. Furthermore, an increase in AP-1 expression promotes the migration, invasion, and metastasis [53]. DNA must be replicated precisely before cell division happens. Defective DNA replication triggers aberrant types of DNA replication, such as DNA re-replication and unscheduled endoreplication, which leads to more aggressive and drug-resistant forms of cancer. The kinases ataxia telangiectasia and rad3-related protein (ATR), ataxia telangiectasia mutated (ATM), and checkpoint kinase 1/2 (CHK1/2) form a crucial DNA damage response module at the stalled replication fork, which is recognized as replication stress. The activation of ATMATR phosphorylates CHK1 and CHK2, which further activates p53 and other downstream molecules as well as the response proteins to replication stress [54].

The GSEA and IPA analysis revealed 23 enriched genes including PDIA4, HDAC1, and MDM2 with higher mutation accumulation scores in the effusionassociated DLBCL samples versus the comparison cohort. PDIA4 has been reported to act as a promoter of tissue factor responsible for coagulation, modulating the function and accumulation of platelets [55]. Stimulated platelets assist in promoting tumor cell metastasis, proliferation, adhesion, and angiogenesis and keep the tumor cells away from the immune system [56]. Thus, PDIA4 might be connection between activated platelets and tumor progression [57]. PDIA2 is a critical prognostic marker that plays an important role in the drugresistance phenotype in ovarian carcinoma [58]. High expression levels of HDAC1 have been reported in diverse cancer types. The expression level of some histone-related proteins including HDAC1, HDAC2, and HDAC6 was significantly higher in cases of DLBCL compared to normal lymphoid tissue. In addition, increased expression of HDAC1 was related to the tumor aggressiveness and a poorer survival in patients with DLBCL [59]. In parallel, we found that overexpression of HDAC1 and MDM2 correlated with the emergence of lymphomatous effusions, and HDAC1 overexpression further predicted worse outcome. The p53 tumor suppressor is negatively regulated by MDM2. p53 
transactivates $M D M 2$, and then MDM2, in turn, degrades and inhibits p53 activity forming a negative feedback loop. MDM2 is reported to be highly expressed in cancers [60]. A noteworthy strategy to activating p53mediated apoptosis in tumors, which overexpress MDM2 and wild-type p53, is to inhibit the interaction of the p53-MDM2 [61]. Our findings suggest that DLBCLassociated lymphomatous effusions may be associated mechanistically with TP53-MDM2 pathway and HDACrelated chromatin remodeling mechanisms.

The weakness of our study is a relatively small number of samples, which is due to the rare occurrence of DLBCL presenting initially with lymphomatous effusions. However, we used two methods, WES and gene microarray, as well as delicate bioinformatics analyses to decipher the genes responsible for the pathogenesis of lymphomatous effusions in DLBCL. Furthermore, we validated our NGS findings with an additional cohort of clinical samples with or without tumorous effusions and confirmed the interesting findings, which warrant further studies with larger, independent cohorts.

\section{Conclusions}

DLBCL is the most common type of lymphoma. A subset of patients may present with lymphomatous effusions initially or during disease progression, and is associated with a poor prognosis of affected patients [4]. In this study we used NGS and GEP to assess effusionbased DLBCL cells and compared the results to noneffusion-associated DLBCL cells. The results showed that effusion-associated DLBCL cells got higher scores in the BCR, NFKB and TLR pathways and in genes responsible for leukocyte migration. On the other hand, IPA highlighted the pivotal role of TP53-related and chromatin remodeling pathways in DLBCL lymphomatous effusions, which were validated on an independent cohort. Our findings have shed light on the prognostic and therapeutic implications for DLBCL patients with lymphomatous effusions.

\footnotetext{
Abbreviations

ATM: ataxia telangiectasia mutated; ATR: ataxia telangiectasia and rad3related protein; BCM: BCL10-CARD11-MALT1; BCR: B-cell receptor; CHK: checkpoint kinase; DLBCL: diffuse large B-cell lymphoma; ES: enrichment score; FAK: focal adhesion kinase; GEP: gene expression profilin; GSEA: Gene Set Enrichment Analysis; ssGSEA: single-sample GSEA; GSVA: Gene Set Variation Analysis; IL: interleukin; IPA: Ingenuity Pathway Analysis; ITAM: immunoreceptor tyrosine-based activation motif; LCL: Iymphoblastoid cell lines; MAF: minor allele frequency; NER: nucleotide excision repair; NGS: next generation sequencing; RMD: regional mutation density; qRT-PCR: quantitative reverse transcription PCR; SNV: single nucleotide variant; indels, insertions/deletions; SUMO: sumoylation; TCGA: The Cancer Genome Atlas; TLR: toll-like receptors; WES: whole exome sequencing; WB: Western blot
}

\section{Supplementary Information}

The online version contains supplementary material available at https://doi. org/10.1186/s40364-021-00330-8.

\section{Additional file 1. \\ Additional file 2. \\ Additional file 3. \\ Additional file 4. \\ Additional file 5 . \\ Additional file 6 .}

\section{Acknowledgements}

Not applicable.

\section{Authors' contributions}

Sina Abdollahi and Seyedeh Zahra Dehghanian: performance of bioinformatic analysis and drafting the article. Liang-Yi Hung and Shiang-Jie Yang: acquisition and analysis of quantitative real-time PCR and immunoblotting data. Dao-Peng Chen: performance of next generation sequencing. L. Jeffrey Medeiros: data analysis and critical review and revision of the draft. Jung-Hsien Chiang and Kung-Chao Chang: design, analysis and interpretation of data and revision of the draft. The author(s) read and approved the final manuscript.

\section{Funding}

This study is supported by grants from the Ministry of Science and Technology, Taiwan (MOST 106-2320-B-006-037-MY3 and MOST 109-2320-B-006-045-MY3), and Kaohsiung Medical University Research Center Grant (KMU-TC109A04-1), Kaohsiung, Taiwan to Dr. KC Chang.

\section{Availability of data and materials}

The data that support the findings of this study are available on request from the corresponding author. WES data are available in the National Center for Biotechnology Information (https://submit.ncbi.nlm.nih.gov/).

\section{Declarations}

\section{Ethical Approval and Consent to participate}

The study was approved by the institutional review board (NCKUH-A-ER-102397 and NCKUH-A-65 ER-105-483) and was in accord with the Helsinki Declaration of 1975, as revised in 2013. Written informed consent has been obtained from the participants involved.

\section{Consent for publication}

Not applicable.

\section{Competing interests}

The authors declare no conflicts of interest.

\section{Author details}

${ }^{1}$ Intelligent Information Retrieval Lab, Department of Computer Science and Information Engineering, National Cheng Kung University, 701 Tainan, Taiwan. ${ }^{2}$ Institute of Biomedical Sciences, National Sun Yat-sen University, Kaohsiung, Taiwan. ${ }^{3}$ Department of Biotechnology and Bioindustry Sciences, College of Bioscience and Biotechnology, National Cheng Kung University, Tainan, Taiwan. ${ }^{4}$ Department of Pharmacology, College of Medicine, National Cheng Kung University, Tainan, Taiwan. ${ }^{5}$ University Center for Bioscience and Biotechnology, National Cheng Kung University, Tainan, Taiwan. ${ }^{6}$ Cancer Molecular Biology and Drug Discovery, College of Medical Science and Technology, Taipei Medical University, Taipei, Taiwan. ${ }^{7}$ Graduate Institute of Medicine, College of Medicine, Kaohsiung Medical University, Kaohsiung, Taiwan. ${ }^{8}$ Institute of Basic Medical Sciences, College of Medicine, National Cheng Kung University, Tainan, Taiwan. ${ }^{9}$ Kim Forest Enterprise Co., Ltd, New Taipei City, Taiwan. ${ }^{10}$ Department of Hematopathology, The University of Texas MD Anderson Cancer Center, Houston, Texas, USA. " Institute of Medical Informatics, National Cheng Kung University, Tainan, Taiwan.

${ }^{12}$ Department of Pathology, College of Medicine, National Cheng Kung University Hospital, National Cheng Kung University, 138 Sheng-Li Road, 704 Tainan, Taiwan. ${ }^{13}$ Department of Pathology, College of Medicine, Kaohsiung Medical University, Kaohsiung, Taiwan. ${ }^{14}$ Department of Pathology, 
Kaohsiung Medical University Hospital, Kaohsiung, Taiwan. ${ }^{15}$ Center for Cancer Research, Kaohsiung Medical University, Kaohsiung, Taiwan.

\section{Received: 27 July 2021 Accepted: 22 September 2021 Published online: 09 October 2021}

\section{References}

1. Chang KC, Huang GC, Jones D, Tsao CJ, Lee JYY, Su IJ. Distribution and prognosis of WHO lymphoma subtypes in Taiwan reveals a low incidence of germinal-center derived tumors. Leuk Lymphoma. 2004;45:1375-84.

2. Das DK. Serous effusions in malignant lymphomas: A review. Diagn Cytopathol. 2006;34:335-47.

3. Mendeville M, Roemer MGM, Van Den Hout MFCM, Los-De Vries GT, Bladergroen $\mathrm{R}$, Stathi $\mathrm{P}$, et al. Aggressive genomic features in clinically indolent primary HHV8-negative effusion-based lymphoma. Blood. 2019;133: $377-80$

4. Chen YP, Huang HY, Lin KP, Medeiros LJ, Chen TY, Chang KC. Malignant effusions correlate with poorer prognosis in patients with diffuse large Bcell Lymphoma. Am J Clin Pathol. 2015;143:707-15.

5. Chapuy B, Stewart C, Dunford AJ, Kim J, Kamburov A, Redd RA, et al. Molecular subtypes of diffuse large $B$ cell lymphoma are associated with distinct pathogenic mechanisms and outcomes. Nat Med. 2018;24:679-90.

6. Schmitz R, Wright GW, Huang DW, Johnson CA, Phelan JD, Wang JQ, et al. Genetics and Pathogenesis of Diffuse Large B-Cell Lymphoma. N Engl J Med. 2018;378:1396-407.

7. Morin RD, Mungall K, Pleasance E, Mungall AJ, Goya R, Huff RD, et al. Mutational and structural analysis of diffuse large B-cell lymphoma using whole-genome sequencing. Blood. 2013;122:1256-65.

8. Pasqualucci L, Trifonov V, Fabbri G, Ma J, Rossi D, Chiarenza A, et al. Analysis of the coding genome of diffuse large B-cell lymphoma. Nat Genet. 2011; 43:830-7.

9. Zhang J, Grubor V, Love CL, Banerjee A, Richards KL, Mieczkowski PA, et al. Genetic heterogeneity of diffuse large B-cell lymphoma. Proc Natl Acad Sci U S A. 2013;110:1398-403.

10. Zhang Y, Xiao Y, Yang M, Ma J. Cancer mutational signatures representation by large-scale context embedding. Bioinformatics. 2020;36:i309-16.

11. Polak P, Karlic R, Koren A, Thurman R, Sandstrom R, Lawrence MS, et al. Cellof-origin chromatin organization shapes the mutational landscape of cancer. Nature. 2015:518:360-4.

12. Gonzalez-Perez A, Sabarinathan R, Lopez-Bigas N. Local Determinants of the Mutational Landscape of the Human Genome. Cell. 2019;177:101-14.

13. Schuster-Böckler B, Lehner B. Chromatin organization is a major influence on regional mutation rates in human cancer cells. Nature. 2012:488:504-7.

14. Collins FS, Guyer MS, Chakravarti A. Variations on a theme: Cataloging human DNA sequence variation. Science. 1997;278:1580-1.

15. Hans CP, Weisenburger DD, Greiner CT, Gascoyne DR, Delabie J, Ott G, et al. Confirmation of the molecular classification of diffuse large B-cell lymphoma by immunohistochemistry using a tissue microarray. Blood. 2004; 103(1):275-82.

16. Sanchez NE, Harty BL, O'Reilly-Pol T, Ackerman SD, Herbert AL, Holmgren M, et al. Whole genome sequencing-based mapping and candidate identification of mutations from fixed zebrafish tissue. G3 Genes. Genomes Genet. 2017;7:3415-25.

17. Shen JC, Kamath-Loeb AS, Kohrn BF, Loeb KR, Preston BD, Loeb LA. A highresolution landscape of mutations in the BCL6 super-enhancer in normal human B cells. Proc Natl Acad Sci U S A. 2019;116:24779-85.

18. Reva B, Antipin $Y$, Sander $C$. Predicting the functional impact of protein mutations: Application to cancer genomics. Nucleic Acids Res. 2011;39(17): e118.

19. Abdollahi S, Lin P-C, Shen M-R, Chiang J-H, WinBinVec: Cancer-Associated Protein-Protein Interaction Extraction and Identification of 20 Various Cancer Types and Metastasis Using Different Deep Learning Models. IEEE Journal of Biomedical and Health Informatics 2021 (in press).

20. Domanska D, Vodák D, Lund-Andersen C, Salvatore S, Hovig E, Sandve GK The rainfall plot: Its motivation, characteristics and pitfalls. BMC Bioinformatics. 2017;18(1):264

21. Abdollahi S, Lin P-C, Shen M-R, Chiang J-H. Precise uncertain significance prediction using latent space matrix factorization models: genomics variant and heterogeneous clinical data-driven approaches. Brief Bioinform. 2020. doi:https://doi.org/10.1093/bib/bbaa281. Online ahead of print.
22. Xu-Monette ZY, Møller MB, Tzankov A, Montes-Moreno S, Hu W, Manyam GC, et al. MDM2 phenotypic and genotypic profiling, respective to TP53 genetic status, in diffuse large B-cell lymphoma patients treated with rituximab-CHOP immunochemotherapy: A report from the International DLBCL Rituximab-CHOP Consortium Program. Blood. 2013;122:2630-40.

23. Marquard L, Poulsen CB, Gjerdrum LM, De Nully Brown P, Christensen IJ, Jensen PB, et al. Histone deacetylase 1, 2, 6 and acetylated histone H4 in Band T-cell lymphomas. Histopathology. 2009;54:688-98.

24. Bonomo L, Feragalli B, Sacco R, Merlino B, Storto ML. Malignant pleural disease. Eur J Radiol. 2000;34:98-118.

25. Huang C, Jacobson K, Schaller MD. MAP kinases and cell migration. J Cell Sci. 2004;117:4619-28.

26. Philip M, Rowley DA, Schreiber H. Inflammation as a tumor promoter in cancer induction. Semin Cancer Biol. 2004;14:433-9.

27. Pan D, Zhu Y, Zhou Z, Wang T, You H, Jiang C, et al. The CBM complex underwrites NF-KB activation to promote HER2-associated tumor malignancy. Mol Cancer Res. 2016:14:93-102.

28. Shchebliakov DV, Logunov DY, Tukhvatulin Al, Shmarov MM, Naroditsky BS, Ginzburg AL. Toll-Like Receptors (TLRs): The Role in Tumor Progression. Acta Naturae. 2010;2:21-9.

29. Huang L, Xu H, Peng G. TLR-mediated metabolic reprogramming in the tumor microenvironment: potential novel strategies for cancer immunotherapy. Cell Mol Immunol. 2018;15:428-37.

30. Xia Y, Shen S, Verma IM. NF-KB, an active player in human cancers. Cancer Immunol Res. 2014;2:823-30.

31. Woyach JA, Johnson AJ, Byrd JC. The B-cell receptor signaling pathway as a therapeutic target in CLL. Blood. 2012;120:1175-84.

32. Koehrer S, Burger JA. B-cell receptor signaling in chronic lymphocytic leukemia and other B-cell malignancies. Clin Adv Hematol Oncol. 2016;1:55-65.

33. Dall'Olio F, Trinchera M. Epigenetic bases of aberrant glycosylation in cancer. Int J Mol Sci. 2017;18(5):998.

34. Magalhães A, Duarte HO, Reis CA. Aberrant Glycosylation in Cancer: A Novel Molecular Mechanism Controlling Metastasis. Cancer Cell. 2017;31:733-5.

35. Oliveira-Ferrer L, Legler K, Milde-Langosch K. Role of protein glycosylation in cancer metastasis. Semin Cancer Biol. 2017:44:141-52.

36. Rowson-Hodel AR, Wald JH, Hatakeyama J, O'Neal WK, Stonebraker JR, VanderVorst $\mathrm{K}$, et al. Membrane Mucin Muc4 promotes blood cell association with tumor cells and mediates efficient metastasis in a mouse model of breast cancer. Oncogene. 2018;37:197-207.

37. Liu Q, Cheng Z, Luo L, Yang Y, Zhang Z, Ma H, et al. C-terminus of MUC16 activates Wnt signaling pathway through its interaction with $\beta$-catenin to promote tumorigenesis and metastasis. Oncotarget. 2016;7:36800-13.

38. Krugmann S, Andrews S, Stephens L, Hawkins PT. ARAP3 is essential for formation of lamellipodia after growth factor stimulation. J Cell Sci. 2006; 119:425-32.

39. Wang QX, Chen ED, Cai YF, Zhou YL, Zheng ZC, Wang YH, et al. Nextgeneration sequence detects ARAP3 as a novel oncogene in papillary thyroid carcinoma. Onco Targets Ther. 2016;9:7161-7.

40. Wu T, Song $H$, Xie D, Zhao B, Xu H, Wu C, et al. Silencing of ASPP2 promotes the proliferation, migration and invasion of triple-negative breast cancer cells via the PI3K/AKT pathway. Int J Oncol. 2018;52:2001-10.

41. Sedgwick AE, D'Souza-Schorey C. Wnt signaling in cell motility and invasion: Drawing parallels between development and cancer. Cancers (Basel). 2016; 8(9):80.

42. Bhattacharya R, Fan F, Wang R, Ye X, Xia L, Boulbes D, et al. Intracrine VEGF signalling mediates colorectal cancer cell migration and invasion. $\mathrm{Br} J$ Cancer. 2017:117:848-55.

43. Yu H, Jove R. The stats of cancer - New molecular targets come of age. Nat Rev Cancer. 2004:4:97-105.

44. Lassmann S, Schuster I, Walch A, Göbel H, Jütting U, Makowiec F, et al. STAT3 mRNA and protein expression in colorectal cancer: Effects on STAT3inducible targets linked to cell survival and proliferation. J Clin Pathol. 2007; 60:173-9.

45. Chang Q, Bournazou E, Sansone P, Berishaj M, Gao SP, Daly L, et al. The IL6/JAK/Stat3 feed-forward loop drives tumorigenesis and metastasis. Neoplasia (United States). 2013;15:848-62.

46. Powell E, Piwnica-Worms D, Piwnica-Worms H. Contribution of p53 to metastasis. Cancer Discov. 2014:4:405-14.

47. Yang M, Huang CZ. Mitogen-activated protein kinase signaling pathway and invasion and metastasis of gastric cancer. World J Gastroenterol. 2015;21: 11673-9. 
48. Yan X, Rui XJ, Zhang K. Baicalein inhibits the invasion of gastric cancer cells by suppressing the activity of the p38 signaling pathway. Oncol Rep. 2015; 33:737-43.

49. Szalat R, Samur MK, Fulciniti M, Lopez M, Nanjappa P, Cleynen A, et al. Nucleotide excision repair is a potential therapeutic target in multiple myeloma. Leukemia. 2018:32:111-9.

50. Gylfe $A E$, Kondelin J, Turunen $M$, Ristolainen $H$, Katainen $R$, Pitkänen $E$, et al. Identification of candidate oncogenes in human colorectal cancers with microsatellite instability. Gastroenterology. 2013;145(3):540-3.e22.

51. Packham S, Warsito D, Lin Y, Sadi S, Karlsson R, Sehat B, et al. Nuclear translocation of IGF-1R via p150Glued and an importin-3/RanBP2dependent pathway in cancer cells. Oncogene. 2015;34:2227-38.

52. Dünnebier T, Bermejo JL, Haas S, Fischer HP, Pierl CB, Justenhoven C, et al. Polymorphisms in the UBC9 and PIAS3 genes of the SUMO-conjugating system and breast cancer risk. Breast Cancer Res Treat. 2010:121:185-94.

53. Leaner VD, Chick JF, Donninger H, Linniola I, Mendoza A, Khanna C, et al. Inhibition of AP-1 transcriptional activity blocks the migration, invasion, and experimental metastasis of murine osteosarcoma. Am J Pathol. 2009;174 265-75.

54. Smith J, Tho LM, Xu N, Gillespie DA. The ATM-Chk2 and ATR-Chk1 Pathways in DNA Damage Signaling and Cancer. Adv Cancer Res. 2010;108: 73-112.

55. Stopa JD, Zwicker Jl. The intersection of protein disulfide isomerase and cancer associated thrombosis. Thromb Res. 2018;164:130-5.

56. Gay LJ, Felding-Habermann B. Contribution of platelets to tumour metastasis. Nat Rev Cancer. 2011:11:123-34.

57. Wang Z, Zhang H, Cheng Q. PDIA4: The basic characteristics, functions and its potential connection with cancer. Biomed Pharmacother. 2020;122: 109688

58. Samanta S, Tamura S, Dubeau L, Mhawech-Fauceglia P, Miyagi Y, Kato H, et al. Expression of protein disulfide isomerase family members correlates with tumor progression and patient survival in ovarian cancer. Oncotarget. 2017:8:103543-56

59. Min SK, Koh YH, Park Y, Kim HJ, Seo J, Park HR, et al. Expression of Hat1 and HDAC1, 2, 3 in diffuse large B-cell lymphomas, Peripheral T-cell lymphomas, and NK/T-cell lymphomas. Korean J Pathol. 2012;46:142-50.

60. Onel K, Cordon-Cardo C. MDM2 and prognosis. Mol Cancer Res. 2004;2(1):1-8.

61. Chène P. Inhibiting the p53-MDM2 interaction: An important target for cancer therapy. Nat Rev Cancer. 2003;3:102-9.

\section{Publisher's Note}

Springer Nature remains neutral with regard to jurisdictional claims in published maps and institutional affiliations.

Ready to submit your research? Choose BMC and benefit from:

- fast, convenient online submission

- thorough peer review by experienced researchers in your field

- rapid publication on acceptance

- support for research data, including large and complex data types

- gold Open Access which fosters wider collaboration and increased citations

- maximum visibility for your research: over $100 \mathrm{M}$ website views per year

At $\mathrm{BMC}$, research is always in progress.

Learn more biomedcentral.com/submissions 